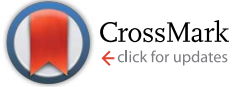

Cite this: RSC Adv., 2017, 7, 15911

\title{
Temperature dependent amplified spontaneous emission of vacuum annealed perovskite films $\uparrow$
}

\author{
Liang Qin, Longfeng Lv, Chunhai Li, Lijie Zhu, Qiuhong Cui, Yufeng Hu, Zhidong Lou, \\ Feng Teng* and Yanbing Hou*
}

Hybrid organic-inorganic halide perovskite crystals exhibit potential applications in amplified spontaneous emission (ASE) devices. The enhancement of the photoluminescence (PL) and full width at half maximum (FWHM) with the temperature dependent phase transition are beneficial to investigate the low threshold of ASE devices. However, the ASE performance and temperature dependent transition characteristics of perovskite films critically depend on morphology and crystallinity. In this paper, we propose vacuumassisted thermal treatment to effectively prepare smooth, condense and well-crystalline $\mathrm{MAPbl}_{3}$ perovskite films. An obvious temperature dependent phase transition occurs in the vacuum annealed $\mathrm{MAPbl}_{3}$ perovskite films, leading to an increase in PL intensity, which could non-monotonically lower the threshold for ASE. Investigating the transient performance at the temperature dependent phase transition underlines an important step in optimizing these novel materials.

Received 25th January 2017

Accepted 28th February 2017

DOI: 10.1039/c7ra01155e

rsc.li/rsc-advances

optically pumped lasing from $\mathrm{MAPbX}_{3}$ perovskite nanocrystals. ${ }^{12,13}$ However, excellent perovskite ASE performance still

\section{Introduction}

Organic-inorganic lead halide perovskites such as $\mathrm{MAPbX}_{3}$ (where $\mathrm{MA}=\mathrm{CH}_{3} \mathrm{NH}_{3}, \mathrm{X}=\mathrm{Cl}, \mathrm{Br}$, and I) have recently emerged as a new class of optoelectronic materials for high-efficiency solar cells, high-intensity light emitting diodes (LEDs) and photodetectors. ${ }^{1-3}$ Owing to their large optical absorption coefficient, high luminescent efficiency, and excellent optical gain, halide perovskites have also attracted considerable attention for their potential application in low-threshold amplified spontaneous emission (ASE) and lasing. ${ }^{4-6}$ Recently, some studies demonstrated that the structure of $\mathrm{MAPbI}_{3}$ crystals were distorted from the tetragonal phase to the orthorhombic phase with a decrease in temperature. ${ }^{7-9}$ Meanwhile, the increase in PL intensity and the decrease in full width at half maximum (FWHM) at a lower temperature are beneficial to the ASE performance, which will allow us to achieve low threshold laser devices. ${ }^{7,10}$ Thus, it is essential to investigate the ASE performance of perovskite materials based on temperature dependent phase transitions.

Some waveguide-based halide perovskite materials have been utilized to achieve optically pumped ASE and lasing. For example, Sum's group obtained wavelength-tunable lasing from $\mathrm{MAPbX}_{3}$ perovskite materials, ${ }^{\mathbf{1 1}}$ Snaith's group achieved a lowthreshold ASE device based on crystal optical reflectors, ${ }^{6}$ and Jin's group and Xiong's group successfully accomplished

Key Laboratory of Luminescence and Optical Information, Ministry of Education, Beijing Jiaotong University, Beijing 100044, China. E-mail: ybhou@bjtu.edu.cn

$\dagger$ Electronic supplementary information (ESI) available: SEM, XRD patterns, ASE performance and TRPL spectra. See DOI: 10.1039/c7ra01155e faces some frustrations, because most narrowed perovskite ASE spectra are sensitive to the crystal structure and waveguide morphology, which may cause the arising scattering loss. ${ }^{\mathbf{1 4}}$ Therefore, detailed ASE properties from bulk perovskite thin films have hardly been investigated, because of the challenges in fabricating thin films with high crystallinity and low roughness.

As an ASE active layer, the well crystallized $\mathrm{MAPbI}_{3}$ film not only provides a platform to analyse the temperature dependent phase transition, but also guarantees the photoluminescence (PL) phenomenon during the temperature dependent phase transition for enhanced ASE performance. ${ }^{\mathbf{1 0 , 1 5}}$ However, conventional methods to prepare perovskite films usually suffer from complex processes as well as low-crystallinity and roughsurface products, which restrict the use of perovskite materials in high-performance ASE applications. Hence, more reliable and facile approaches are increasingly demanded to rationally control the morphology properties and crystallinity characteristics of perovskite films.

In this paper, we demonstrated that vacuum-assisted thermal treatment can reduce pores and improve crystallinity, resulting in an enhanced ASE performance of the $\mathrm{MAPbI}_{3}$ perovskite thin film. Meanwhile, the highly ordered perovskite thin film exhibits an obvious temperature dependent phase transition proved by the change in the PL spectra and XRD pattern, which allows us to investigate the low temperature ASE performance. An abnormal ASE threshold changing nonmonotonically with temperature was observed for the first time. These results provide an important step forward by enhancing 
the performance of perovskite-based ASE and give us a full understanding of these novel materials.

\section{Results and discussion}

Vacuum-assisted thermal treatment (Fig. 1(a)) was used to prepare the pore-free $\mathrm{MAPbI}_{3}$ films. In the fabrication process, a vacuum chamber was utilized for vacuum-assisted annealing of the samples at $90{ }^{\circ} \mathrm{C}$ after spin coating the mixture of perovskite precursor solution onto a glass substrate. For comparison, samples were thermally annealed in $\mathrm{N}_{2}$ and in a vacuum, separately. The thin film formed via annealing in $\mathrm{N}_{2}$ presented a porous and rough morphology as shown in Fig. 1(b). However, a perovskite thin film with a large grain size and smooth surface was achieved by annealing in a vacuum (Fig. 1(c)). As is well known, DMSO possesses a high boiling point $\left(189{ }^{\circ} \mathrm{C}\right)$ and low volatility at room-temperature. It is difficult to get dry perovskite films during the spin coating process. Hence, the wet $\mathrm{MAPbI}_{3}$ films will continually assemble and crystallize, which leads to a porous and rough morphology. Yet by adopting the vacuum chamber for thermal annealing, in terms of the lower saturated vapor pressure $\left(1.3 \mathrm{kPa}\right.$ at $\left.70{ }^{\circ} \mathrm{C}\right)$, DMSO is easy to evaporate under vacuum conditions, which allows the $\mathrm{MAPbI}_{3}$ molecule to assemble into a well crystallized structure. ${ }^{16,17}$ The X-ray diffraction (XRD) patterns in Fig. 1(d) verified that the crystallinity of the $\mathrm{MAPbI}_{3}$ films is improved after annealing in vacuum.
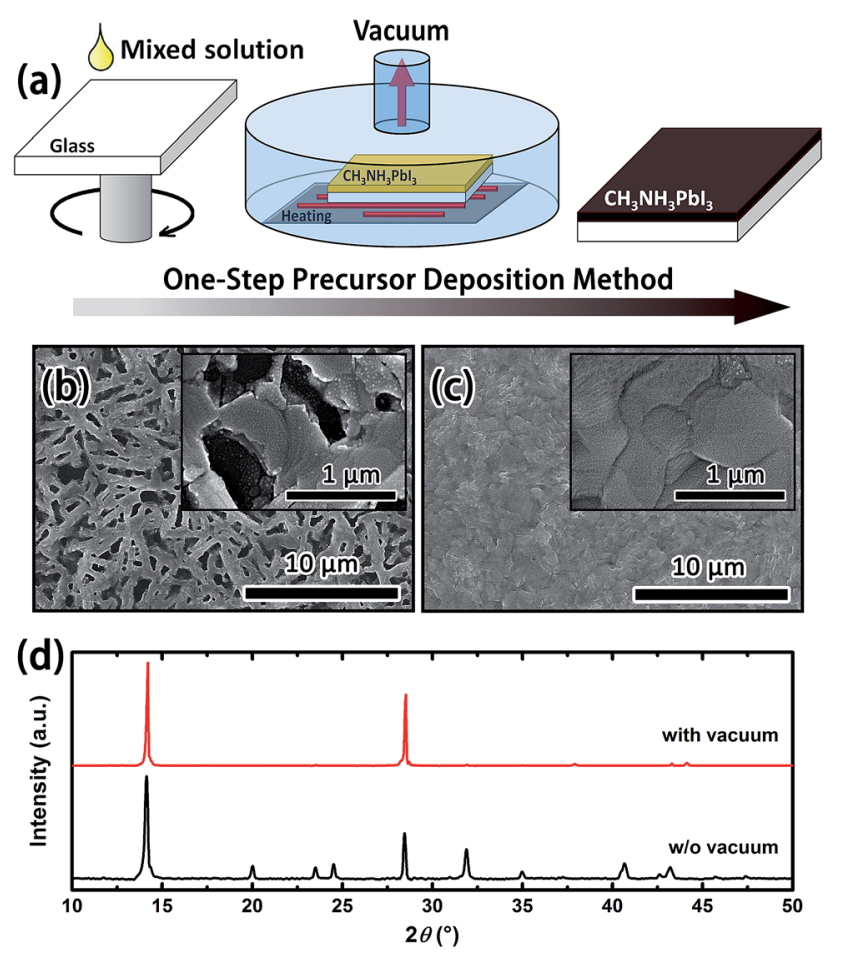

Fig. 1 (a) Scheme of the vacuum-assisted deposition process. The SEM images of MAPbl films formed through annealing in $\mathrm{N}_{2}$ (b) and under vacuum-assistance (c). (d) The XRD patterns of $\mathrm{MAPbl}_{3}$ films annealed in $\mathrm{N}_{2}$ and vacuum.
The morphology and crystallinity of the films treated under vacuum for different lengths of time were investigated by SEM images (Fig. S1 $\dagger$ ) and XRD patterns (Fig. S2 $\dagger$ ). The results indicate that more dense and smooth films were obtained by vacuuming for more than $10 \mathrm{~min}$. The ASE performances of the corresponding films (Fig. S3†) were observed after vacuumannealing with the setup shown in Fig. S4 $\uparrow$ and the lowest threshold was $\sim 40 \mu \mathrm{J} \mathrm{cm}{ }^{-2}$, which is a good result from perovskite based thin films. ${ }^{\mathbf{1 0}, 11}$ To further exhibit the optimization through vacuum-annealing, the ASE net gain and loss coefficients were studied and are shown in Fig. S5. $\dagger$ The increasing net gain and decreasing loss coefficients of each sample are summarized in Table $\mathrm{S} 1, \dagger$ which confirms the improved quality of the $\mathrm{MAPbI}_{3}$ films by the increase in vacuuming time.

As previously reported, the crystal structure of $\mathrm{MAPbI}_{3}$ can transform from the tetragonal phase to the orthorhombic phase with decreasing temperature. ${ }^{\mathbf{1 8}}$ The temperature dependent phase transition can obviously affect the optical properties of $\mathrm{MAPbI}_{3}$ films. The PL spectra shown in Fig. S6† show that the temperature dependent mutation obviously occurred in the perovskite film with optimized morphology and crystallinity and was described as the temperature dependent phase transition. ${ }^{7}$ The above results demonstrate that vacuum-assisted annealing can eliminate pores and improve the crystallinity of perovskite thin films, enhancing the ASE performance and temperature dependent transition significantly. All the following investigations were based on the optimized $\mathrm{MAPbI}_{3}$ perovskite film with the morphology as shown in Fig. 1(c).

The temperature dependent XRD measurement was taken to provide direct evidence of the crystal structure phase transition, as shown in Fig. 2. The XRD diffraction peaks reveal good crystallinity and uniform orientation, which confirms the formation of the orthorhombic and the tetragonal perovskite structures. ${ }^{9,19}$ Above $150 \mathrm{~K}$, the strong characteristic XRD peaks of the tetragonal phase at $14.126^{\circ}, 23.521^{\circ}, 28.463^{\circ}$ and $31.895^{\circ}$

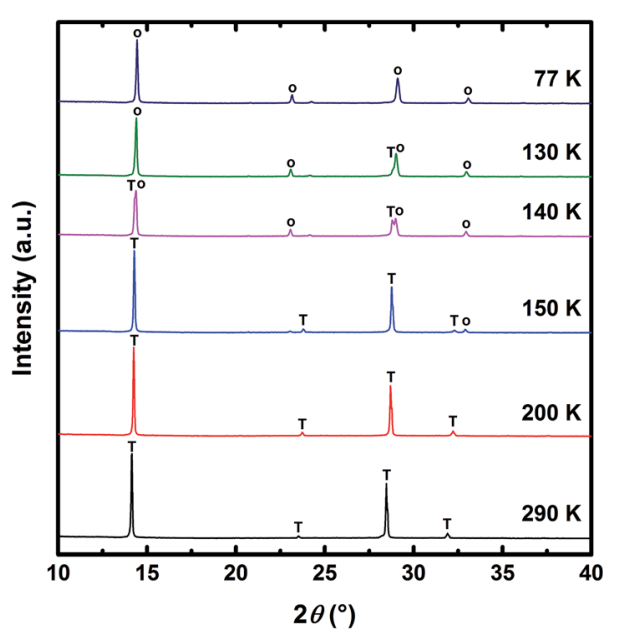

Fig. 2 The XRD patterns of the optimized MAPbl 3 perovskite film at various temperatures. $T$ and $O$ are $X R D$ peaks from the tetragonal phase and orthorhombic phase respectively. 
were observed. At $150 \mathrm{~K}$, another diffraction peak emerges at $32.895^{\circ}$. With a further decrease in temperature, strong characteristic peaks at $14.371^{\circ}, 23.071^{\circ}, 28.994^{\circ}$ and $32.936^{\circ}$ confirm that the crystal structure of perovskite film transitions to the orthorhombic structure.

To clearly investigate the change in crystal structure, we zoom in on the characteristic XRD peaks in Fig. $S 7(a) \dagger$ and summarize the peak intensity and peak position in Fig. $S 7$ (b).$\dagger$ At the beginning of cooling, the change in the peak intensity and the peak position may come from the lattice contraction of $\mathrm{MAPbI}_{3}$. Around $150 \mathrm{~K}$, the coexistence of the orthorhombic phase and the tetragonal phase inhibits the diffraction intensity and changes the XRD peak position abruptly, which is attributed to the random crystal orientation and distorted lattice structure. When the temperature continually decreases, the characteristic peaks of the tetragonal phase are hardly observed. Our XRD results clearly confirm the temperature dependent crystal structure transition between the tetragonal phase and the orthorhombic phase in the well crystalline $\mathrm{MAPbI}_{3}$ perovskite films.

Due to the temperature dependent phase transition, the optical absorption spectra of the optimized $\mathrm{MAPbI}_{3}$ perovskite film were closely related to the temperature, as shown in Fig. 3. The thin film exhibits ordinary absorption spectra above $270 \mathrm{~K}$. A sharp shoulder at $\sim 770 \mathrm{~nm}$ emerges at lower temperatures, which is caused by an excitonic transition, and a second shoulder emerges at $\sim 740 \mathrm{~nm}$ below $150 \mathrm{~K} .{ }^{20}$ With a further decrease in temperature, the latter shoulder $(\sim 740 \mathrm{~nm})$ gains strength in intensity and shifts to a longer wavelength while the first shoulder $(\sim 770 \mathrm{~nm})$ disappears, which can be attributed to the phase transition of the $\mathrm{MAPbI}_{3}$ thin films from a tetragonal phase to an orthorhombic phase, caused by continuous expansion and shrinkage of the lattice. ${ }^{\mathbf{2 1 , 2 2}}$ The exciton binding energy $\left(E_{\mathrm{B}}\right)$ can be calculated using the temperature-dependent absorption line width of the excitonic transition as described in ref. 20. By fitting the absorption data, we estimate an exciton binding energy of $45 \pm 13 \mathrm{meV}$ in the optimized $\mathrm{MAPbI}_{3}$ film, which is shown in Fig. S8. $\dagger$

PL performances of the perovskite film during the phase transition are investigated and presented in Fig. 4. The deconvoluted Gaussian peaks of the PL emission band, illustrated by the colored curves at typical temperatures in Fig. 4(a), clearly

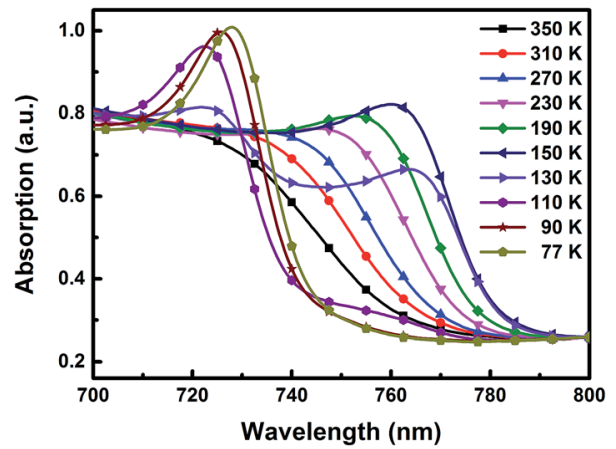

Fig. 3 The absorption spectra of the optimized perovskite film at various temperatures (350-77 K).

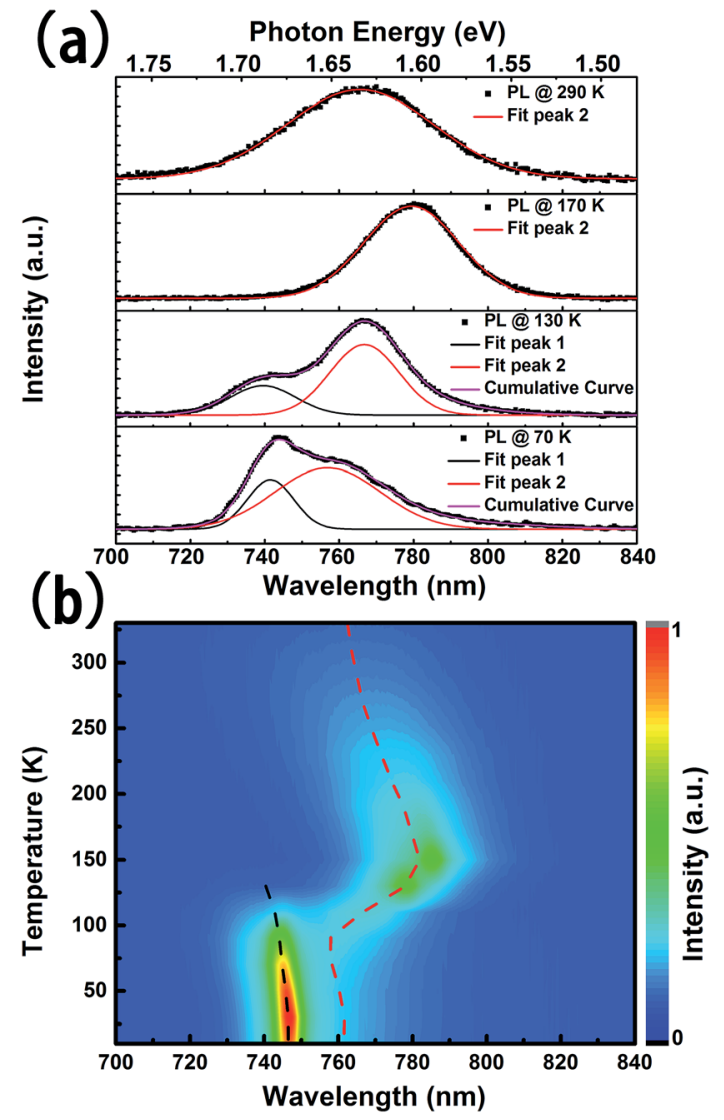

Fig. 4 (a) The PL spectra and fitted peaks at typical temperatures of the optimized perovskite film. (b) The peak position is plotted as the dashed line in the image of the temperature dependent PL spectra in the temperature range from $350 \mathrm{~K}$ to $10 \mathrm{~K}$.

show that the emission spectrum depends on temperature. The PL peak at room temperature $(290 \mathrm{~K})$, named peak 2, of the $\mathrm{MAPbI}_{3}$ perovskite film centers at $\sim 770 \mathrm{~nm}$. With decreasing temperature, a red shift of peak 2 is observed. In the range of 130-10 K, the second PL peak, named peak 1, at a shorter wavelength arises and is enhanced. The temperature dependent PL spectra are mapped on the normalized false color image in Fig. 4(b), where the black dotted line and red dotted line mark the positions of the deconvoluted peaks of the PL spectra at different temperatures. In the range of 350-160 K, peak 2 shows a red shift and intensity increase with decreasing temperature, which is due to the reduction in the dissociation of free excitons and the change in material parameters contrasted to typical semiconductors. ${ }^{7,22}$ When further decreasing the temperature, an abnormal blue shift of peak 2 was observed in the range of $150 \mathrm{~K}$ to $100 \mathrm{~K}$. Meanwhile, a new peak (peak 1) appears at $\sim 740 \mathrm{~nm}$ and the intensity gradually becomes strong, which is attributed to the phase transition from the tetragonal phase to the orthorhombic phase., ${ }^{9,18}$ Peak 1 and peak 2 are traced to the tetragonal phase and orthorhombic phase of $\mathrm{MAPbI}_{3}$, respectively. Furthermore, by reducing the temperature to $10 \mathrm{~K}$, the central wavelength of the PL spectra and the intensity of peak 1 increased continually. The change of the PL peak positions is in accordance with the observation of the absorption edge of 
$\mathrm{MAPbI}_{3}$ at various temperatures (Fig. 3), which is a striking indication of the crystal structural phase transition. ${ }^{21}$

Based on the investigation of temperature dependent PL spectra, we know that the PL efficiency is enhanced with decreasing temperature, which is beneficial for lowering the ASE threshold. ${ }^{23}$ Fig. 5(a) presents the emission spectra of the perovskite thin film under $500 \mathrm{~nm}$ excitation with various pump intensities. Under low power excitation, only a broad spontaneous emission (SE) band can be observed at the $\sim 780 \mathrm{~nm}$ central wavelength. With gradually increasing pump intensity, the FWHM slowly decreases. When the pump intensity is above the threshold, ASEs for the orthorhombic phase and the tetragonal phase arise at about $\sim 770 \mathrm{~nm}$ and $\sim 740 \mathrm{~nm}$, respectively. When the temperature is around $130 \mathrm{~K}$, where the orthorhombic phase and the tetragonal phase coexist, ASE happens in the orthorhombic phase, although PL is more intense in the tetragonal phase.

To characterize the ASE performances at the different temperatures quantitatively, we calculated the ASE threshold for different temperatures. The dependence of output emission on pump intensity at the typical temperatures is shown in Fig. 5(b). From this figure, one can find that the ASE intensity is enhanced and the threshold is reduced by decreasing temperature. The threshold, which is $\sim 40 \mu \mathrm{J} \mathrm{cm}^{-2}$ at room temperature, has reached its lowest, $\sim 10 \mu \mathrm{J} \mathrm{cm}^{-2}$, around $50 \mathrm{~K}$, which is roughly the same as the resonance induced ASE performance based on the perovskite materials excited by a femtosecond laser $(\sim 100 \mathrm{fs}){ }^{6,13}$ However, a non-monotonic rise of the threshold and abnormal peak shift are observed at about $130 \mathrm{~K}$, which may come from the co-existence of the tetragonal phase and the orthorhombic phase. The messy crystal orientation leads to the increase in scatter in the film. ${ }^{\mathbf{1 0}}$ The characters of SE and ASE at each temperatures are summarized in Fig. 5(c).
Strikingly, the shift of the SE peaks with decreasing temperatures is in accordance with that of the PL spectra in Fig. 4(b). In this work, the position of the ASE peak was investigated. The ASE appears at the longer wavelength side of the PL peak above $130 \mathrm{~K}$, and rises at the shorter wavelength side below $130 \mathrm{~K}$, because of the narrower band gap of tetragonal $\mathrm{MAPbI}_{3} \cdot{ }^{1924}$ The increase in band gap results in the reduction in self-absorption at the shorter wavelength side.

To understand the abnormal increase of the ASE threshold and the ASE peak shifting, the mapping of time-resolved PL spectra at different temperatures was investigated in Fig. 6(a). From mapping, we find that the lifetime of the peak from the orthorhombic phase is shorter than that from the tetragonal phase. As is well known, the excited states with longer lifetimes will easily create population inversion, which benefits the ASE performance. Fig. 6(b) summarizes the temperature dependence and the lifetimes of the emissions from orthorhombic and tetragonal $\mathrm{MAPbI}_{3}$. Notably, the lifetimes of each PL peak all decrease to a lower level at $\sim 140 \mathrm{~K}$, resulting from the dispersion of the carriers. ${ }^{9}$ The energy transfer and recombination processes in the coexisting phases can be supported by the Time-Resolved PL (TRPL) spectra shown in Fig. 6(c), where the dashed lines denote the shift of the emission peaks. From the TRPL spectra, peak 2 shifts to a longer wavelength with increasing delay time, which means that the carriers gradually migrate to a lower energy band after pulsed excitation. This redshifted PL emission was attributed to a donor-acceptor pair (DAP) recombination. ${ }^{9}$ The photon energy and the recombination rate of the DAP emission are described by the following equations:

$$
\hbar \omega=E_{\mathrm{g}}-E_{\mathrm{A}}-E_{\mathrm{D}}+e^{2} /\left(4 \pi \varepsilon_{0} \varepsilon R\right)
$$
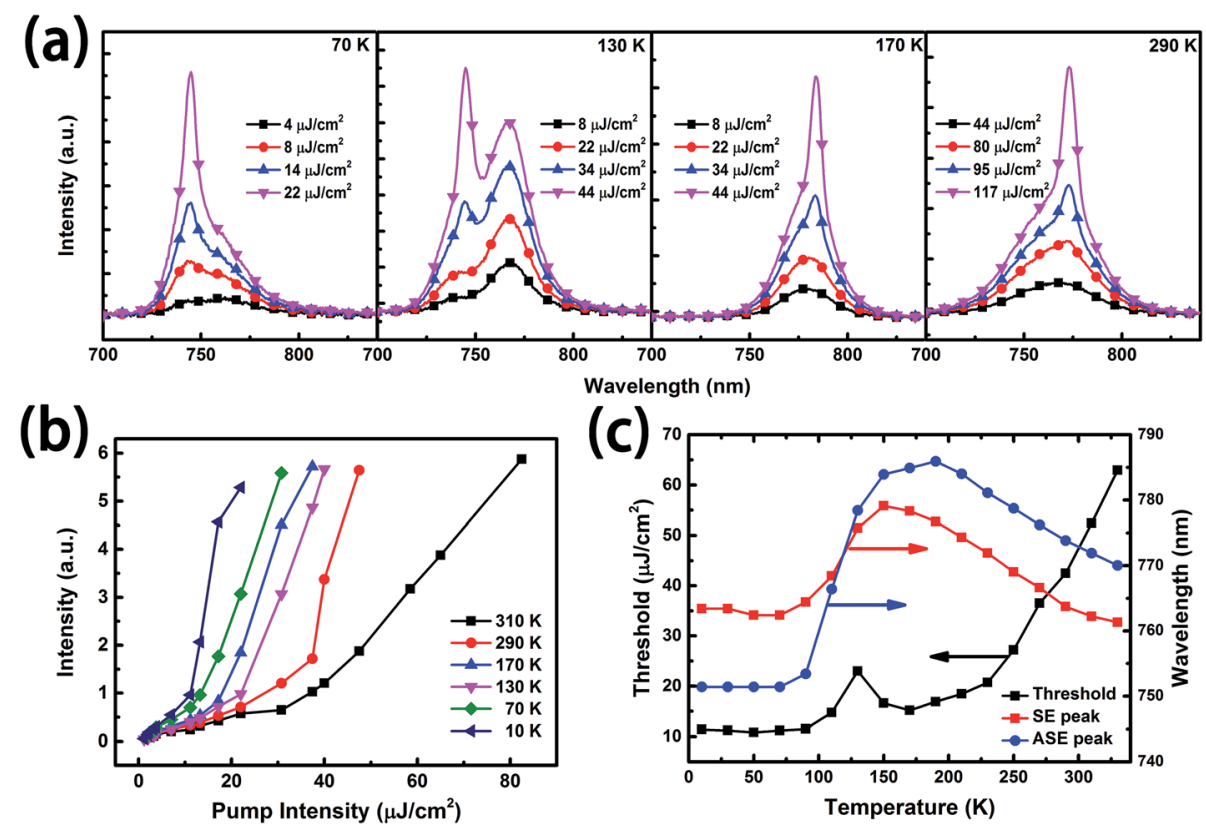

Fig. 5 (a) The PL spectra of the MAPb/ film at different excitation intensities and temperatures. (b) The output intensity as a function of pump intensity at different temperatures. (c) The temperature dependence of the PL peak position, ASE peak position and ASE threshold. 

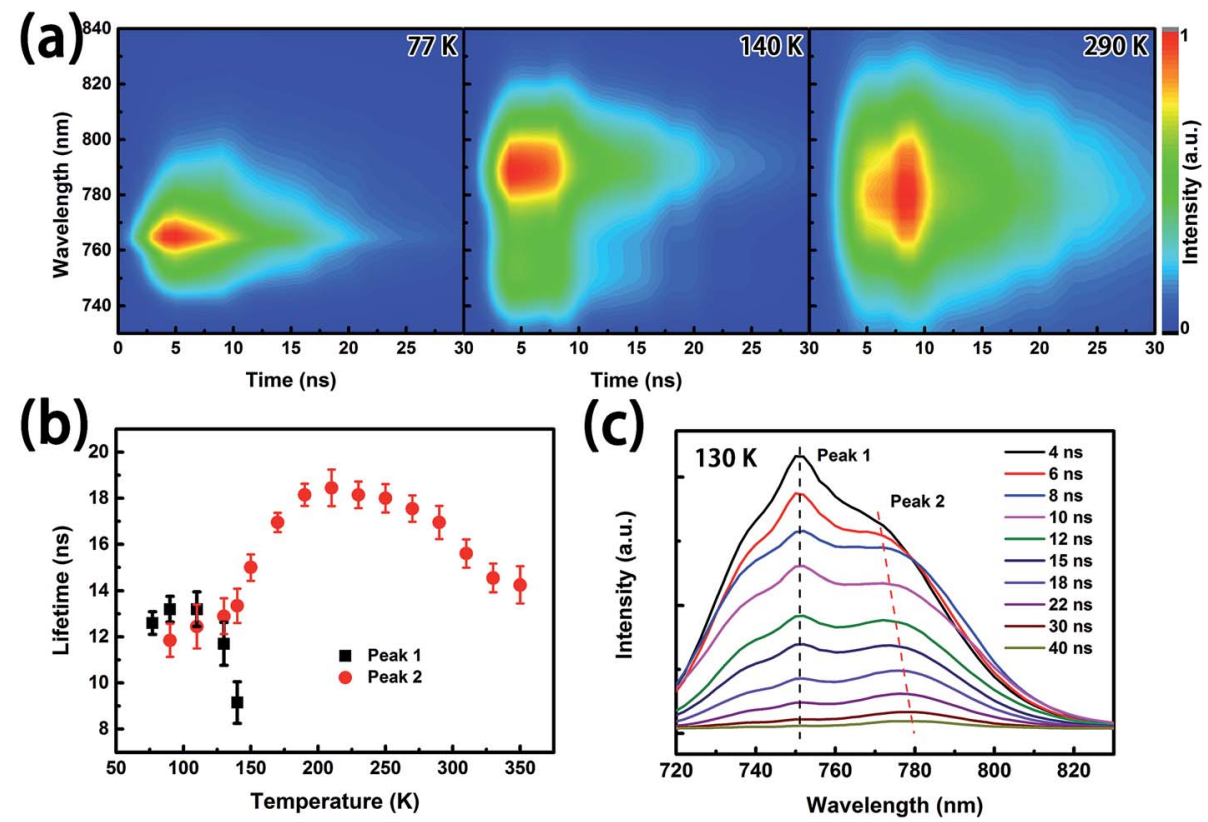

Fig. 6 (a) Wavelength dependent PL lifetime mapping at $77 \mathrm{~K}, 140 \mathrm{~K}$ and $290 \mathrm{~K}$. The effect of temperature on the lifetime of each PL peak (b) and the time resolution PL spectra as a function of time (c).

$$
1 / \tau_{\mathrm{DA}} \propto \exp \left[-2\left(R / \alpha_{\mathrm{D}, \mathrm{A}}\right)\right]
$$

where $E_{\mathrm{g}}$ is the band-gap energy, $\varepsilon_{0}$ is the static dielectric constant and $E_{\mathrm{D}}$ and $E_{\mathrm{A}}$ are the donor and acceptor binding energies, respectively. $R$ is the distance between ionized donors and acceptors. $1 / \tau_{\mathrm{DA}}$ and $\alpha_{\mathrm{D}, \mathrm{A}}$ are the radiative recombination rate and the larger Bohr-radius equivalent of the donor and acceptor, respectively. From the equations, the emission energy $(\hbar \omega)$ from the spatially close pairs is higher than that from the spatially distant pairs, due to the larger Coulomb interactions. Hence, the recombination rate for the high-energy transition is larger than that for the low-energy transition, which results in faster decay times for the photons with shorter wavelengths in the PL band. According to the lifetime of each peak (Fig. 6(b)), we can deduce that the red-shift of peak 2 at $\sim 140 \mathrm{~K}$ is a gradual transition from near-band-edge recombination at the tetragonal phase to DAP recombination at the orthorhombic phase. ${ }^{9}$ Our XRD and optical measurements at low temperature clearly reveal that the tetragonal phase and the orthorhombic phase can co-exist in a broad temperature range. Normally, the co-existence of two phases would form multiple sub-band energy levels and induce an increase in non-radiative decay, which leads to a frustration of the ASE threshold. ${ }^{9,19,24}$ This is the first time that the abnormal change of the ASE threshold performance, based on the $\mathrm{MAPbI}_{3}$ perovskite thin film, during temperature based phase transition has been noticed and investigated.

\section{Conclusions}

In summary, vacuum-assisted annealing has been used in the fabrication of dense and smooth $\mathrm{MAPbI}_{3}$ films. The crystallinity and morphology are improved by increasing the vacuum time before annealing the films. The optimized sample exhibits excellent ASE performance and enhanced temperature dependent phase transition, which proves that the vacuum-assisted one-step precursor deposition method is an effective strategy for the fabrication of well-controlled morphology and crystallinity. The temperature dependent crystal structure phase transition was observed and proved by XRD patterns, absorption spectra and PL spectra with decreasing temperature. In addition, we have also demonstrated the ASE performance during the phase transition. The ASE performance was wellenhanced by decreasing the temperature and influenced by the phase transition. Our results, showing enhanced ASE performance with the lowest threshold of $\sim 40 \mu \mathrm{J} \mathrm{cm}^{-2}$ at room temperature and $\sim 10 \mu \mathrm{J} \mathrm{cm}^{-2}$ at about $50 \mathrm{~K}$, clearly indicate that the vacuum-assisted precursor deposition method can enhance the temperature dependent optical characteristics, and provides an excellent pathway for achieving high-performance and cost-effective perovskite-based coherent light source devices that can be implemented in the near future.

\section{Experimental section}

\section{Sample preparation}

Condense and pore-free perovskite films were prepared via a vacuum-assisted thermal treatment. The mixture of $\mathrm{PbI}_{2}$ (99\%, Alfa Aesar) and MAI (99\%, Dyesol) in a stoichiometric ratio was dissolved in DMSO (99.8\%, Alfa Aesar) with a concentration of $0.8 \mathrm{M}$ and stirred at $70{ }^{\circ} \mathrm{C}$ overnight in a nitrogen filled glove box, and cooled down to $50{ }^{\circ} \mathrm{C}$ before spin-coating. The perovskite layer was then deposited by spin coating the precursor solutions directly on the glass substrates at $3000 \mathrm{rpm}$ for $50 \mathrm{~s}$. After spin coating, the films were immediately vacuumed in a chamber for 0, 5, $10 \mathrm{~min}$ and more without annealing. Then the perovskite films with a typical 
thickness of $350 \mathrm{~nm}$ were obtained by heating the films to $90{ }^{\circ} \mathrm{C}$ slowly $\left(\sim 1.5{ }^{\circ} \mathrm{C} \min ^{-1}\right)$ and keeping for $4 \mathrm{~h}$ under vacuum.

\section{Film characterization}

A field emission scanning electron microscope (FE-SEM, Hitachi-S4800) was used to obtain SEM images. The room temperature X-ray diffraction (XRD) patterns were recorded by a Bruker D8 Advance X-ray diffractometer. The temperature dependent XRD patterns were measured by a Bruker D8 Discover X-ray diffractometer with non-ambient stages. The thicknesses of the films were measured by an Ambios Technology XP-2 surface profilometer. The temperature dependent Ultraviolet-Visible (UV-vis) absorption spectra were collected by a Shimadzu UV-3101PC spectrometer with a Microscopy Cryostat System (Cryo Industries of America). A Continuum SureLite II Nd3+: YAG laser pumping a Continuum SureLite optical parametric oscillator (OPO) was employed as the excitation source for ASE and TRPL measurements of the perovskite films. The laser pulse energy was detected with a Coherent J-10SI-HE power meter. The temperature dependent ASE emission spectra were recorded using an Acton-150 imaging spectrophotometer with a Helium Cryostat System (Oxford Optistat, CCC1104). The temperature dependent PL spectra were collected by a spectrometer (Horriba, iHR320). The TRPL spectra were acquired by a spectrometer (Jobin Yvon Triax 320). The schematic of the optical measurement setup is shown in Fig. S4. $\dagger$

\section{Acknowledgements}

This research is supported by the Natural Science Foundation of China (Grant No. 61475017, 61275175, 61377028, 61475014, 61675018, 61674012 and 51603010), and the Fundamental Research Funds for the Central University (2014JBZ009).

\section{Notes and references}

1 W. S. Yang, J. H. Noh, N. J. Jeon, Y. C. Kim, S. Ryu, J. Seo and S. I. Seok, Science, 2015, 348, 1234-1237.

2 H. Cho, S.-H. Jeong, M.-H. Park, Y.-H. Kim, C. Wolf, C.-L. Lee, J. H. Heo, A. Sadhanala, N. Myoung, S. Yoo, S. H. Im, R. H. Friend and T.-W. Lee, Science, 2015, 350, 1222-1225.

3 L. Dou, Y. M. Yang, J. You, Z. Hong, W. H. Chang, G. Li and Y. Yang, Nat. Commun., 2014, 5, 5404.

4 F. Deschler, M. Price, S. Pathak, L. E. Klintberg, D. D. Jarausch, R. Higler, S. Huttner, T. Leijtens,
S. D. Stranks, H. J. Snaith, M. Atature, R. T. Phillips and R. H. Friend, J. Phys. Chem. Lett., 2014, 5, 1421-1426.

5 G. Xing, N. Mathews, S. Sun, S. S. Lim, Y. M. Lam, M. Gratzel, S. Mhaisalkar and T. C. Sum, Science, 2013, 342, 344-347.

6 S. D. Stranks, S. M. Wood, K. Wojciechowski, F. Deschler, M. Saliba, H. Khandelwal, J. B. Patel, S. J. Elston, L. M. Herz, M. B. Johnston, A. P. H. J. Schenning, M. G. Debije, M. K. Riede, S. M. Morris and H. J. Snaith, Nano Lett., 2015, 15, 4935-4941.

7 K. Wu, A. Bera, C. Ma, Y. Du, Y. Yang, L. Li and T. Wu, Phys. Chem. Chem. Phys., 2014, 16, 22476-22481.

8 X. Y. Chin, D. Cortecchia, J. Yin, A. Bruno and C. Soci, Nat. Commun., 2015, 6, 7383.

9 W. Kong, Z. Ye, Z. Qi, B. Zhang, M. Wang, A. Rahimi-Iman and H. Wu, Phys. Chem. Chem. Phys., 2015, 17, 16405-16411.

10 T. S. Kao, Y.-H. Chou, C.-H. Chou, F.-C. Chen and T.-C. Lu, Appl. Phys. Lett., 2014, 105, 231108.

11 G. Xing, N. Mathews, S. S. Lim, N. Yantara, X. Liu, D. Sabba, M. Gratzel, S. Mhaisalkar and T. C. Sum, Nat. Mater., 2014, 13, 476-480.

12 H. Zhu, Y. Fu, F. Meng, X. Wu, Z. Gong, Q. Ding, M. V. Gustafsson, M. T. Trinh, S. Jin and X. Y. Zhu, Nat. Mater., 2015, 14, 636-642.

13 Q. Zhang, S. T. Ha, X. Liu, T. C. Sum and Q. Xiong, Nano Lett., 2014, 14, 5995-6001.

14 L. Qin, L. Lv, Y. Ning, C. Li, Q. Lu, L. Zhu, Y. Hu, Z. Lou, F. Teng and Y. Hou, RSC Adv., 2015, 5, 103674-103679.

15 Y. Yasuhiro, N. Toru, E. Masaru, W. Atsushi and K. Yoshihiko, Appl. Phys. Express, 2014, 7, 032302.

16 Z. Xiao, Q. Dong, C. Bi, Y. Shao, Y. Yuan and J. Huang, Adv. Mater., 2014, 26, 6503-6509.

17 M. Nishimura, M. Nakayama and T. Yano, J. Chem. Eng. Jpn., 1972, 5, 223-226.

18 A. Poglitsch and D. Weber, J. Chem. Phys., 1987, 87, 6373.

19 T. Baikie, Y. Fang, J. M. Kadro, M. Schreyer, F. Wei, S. G. Mhaisalkar, M. Graetzel and T. J. White, J. Mater. Chem. A, 2013, 1, 5628-5641.

20 V. D'Innocenzo, G. Grancini, M. J. Alcocer, A. R. Kandada, S. D. Stranks, M. M. Lee, G. Lanzani, H. J. Snaith and A. Petrozza, Nat. Commun., 2014, 5, 3586.

21 T. Ishihara, J. Lumin., 1994, 60, 269-274.

22 Y. P. Varshni, Physica, 1967, 34, 149-154.

23 R. Gupta, J. Park, V. Srdanov and A. Heeger, Synth. Met., 2002, 132, 105-107.

24 Y. Wang, T. Gould, J. F. Dobson, H. Zhang, H. Yang, X. Yao and H. Zhao, Phys. Chem. Chem. Phys., 2014, 16, 1424-1429. 